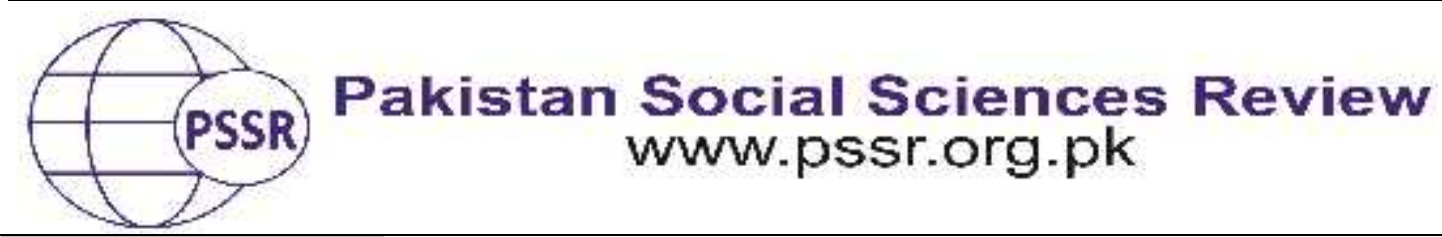

RESEARCH PAPER

\title{
Investigating the Efficacy of Discrete Trial Training Program in the Development of Sustained Eye Contact in an Adolescent Girl with Autism Spectrum Disorder: A Case Study
}

\author{
Hina Hadayat Ali ${ }^{1}$ Dr. Hina Fazil 2
}

1. Ph. D Scholar, Department of Special Education, University of the Punjab, Lahore, Punjab, Pakistan.

2. Assistant Professor, Department of Special Education, University of the Punjab, Lahore, Punjab, Pakistan

\begin{tabular}{|c|c|}
\hline PAPER INFO & ABSTRACT \\
\hline Received: & The current study focused on investigating the efficacy of discrete \\
\hline February 21, 2020 & trial training (DTT) program in development of sustained eye \\
\hline Accepted: & contact in children with ASD. The single subject study design in \\
\hline March 15, 2020 & this research was based on A-B-C-D-E-A and two follow up \\
\hline $\begin{array}{l}\text { Online: } \\
\text { March 30, } 2020\end{array}$ & periods. The participant was the minimal verbal adolescent girl \\
\hline Keywords: & observed on DSM-5 by the psycho \\
\hline Autism Spectrum & researchers found her major area of deficit by using self- \\
\hline & developed rating scale for the identi \\
\hline Discrete Trial & communication deficits of children with ASD which was \\
\hline Training, & validated by experts. DTT was then manipulated over developing \\
\hline Sustained Eye & social communication skills under four different contexts taken as \\
\hline Contact & treatment conditions of the experiment. Efficacy of DTT \\
\hline Corresponding & cved under triad analysis was found comparatively higher \\
\hline Author & $\begin{array}{l}\text { during E phase of treatment. It was recommended that furnished } \\
\text { autism friendly training rooms should be established by the }\end{array}$ \\
\hline high786aims@g & $\begin{array}{l}\text { Punjab government to gain maximum advantages of the DTT } \\
\text { program. }\end{array}$ \\
\hline
\end{tabular}

\section{Introduction}

Children with autism spectrum disorder (ASD) have social communication deficits (American Psychiatric Association, 2013).Social communication deficits may include failing to respond to name, resisting to cuddle, resisting to hold, delayed speech, abnormal voice tone, repeating words or phrases, abnormal conversational patterns, inappropriate social greetings including social initiations and social responding, impaired eye contact, impaired facial expressions, impaired expression of feelings and emotions, impaired sharing of interests, impaired gestures, impaired recognition of non-verbal social cues and others. 
Various social communication deficits have been efficiently developed under the structured format of DTT across the world e.g. teaching to increase social interactions and social initiations (Garfinkle \& Schwartz, 2002; Garcia-Albea et al., 2014; Groskreutz et al., 2015), teaching to offer help to others (Harris et al., 1990; Reeve et al., 2007), teaching to share (Marzullo-Kerth et al., 2011), teaching empathy skills (Schrandt et al., 2009), teaching affective behavior (Gena et al., 2005; DeQuinzio et al., 2007), teaching perspective taking skills (LeBlanc et al., 2003), and teaching to increase joint attention (Kasari et al., 2006; Jones et al., 2006; Krstovska-Guerrero \& Jones, 2013). Jennett et al. (2008) explained that eye contact in three participants was a bit better under the format of discrete trial instruction.

The study conducted by (Jannet et al., 2008) was a comparative study which targeted to make requests and found eye contact slightly better during DTT condition. Notably, this study did not directly target to develop sustained eye contact in children with ASD. This scenario leaves a gap to be fulfilled by manipulating DTT variable over developing social communication among children with ASD skills taken as dependent variable in Pakistani society.

Above mentioned existing literature shows the efficacy of DTT in developing social communication skills across the world on one hand and highlights no piece of research on the variables of the current research to be studied in Pakistani society on other hand. This scenario again leaves a gap to be fulfilled by manipulating DTT variable over developing social communication among children with ASD skills taken as dependent variable in Pakistani society.

Amongst the above mentioned literature, developing sustained eye contact is of utmost importance to achieve other social communication skills. As this skill has an atypical pattern in children with ASD (Senju et al., 2003). Therefore, children with ASD avoid eye contact which makes social interaction a challenge for this segment of population and ultimately need to be intervened for this fundamental skill.

Therefore, underlying impaired eye contact in children with ASD may be dealt with systematically designed intensive and early intervention strategies. By executing highly structured patterns of intervention, this segment of population may develop sustained eye contact specifically and enable them to communicate socially in an appropriate way generally. DTT is one of the one-on-one intensive teaching methods in the treatment of ASD (Smith, 2001).

Finally, developing sustained eye contact in children with ASD under the systematic format of DTT has not been researched in Pakistan till now. This forced the researchers to plan and execute DTT to investigate its efficacy in the development of sustained eye contact for an adolescent child with ASD in Pakistani society. As Hamdan (2018) also proposed discrete trial training program to develop non-verbal communication skills in children with ASD, and sustained eye contact is one of the crucial universal types of non-verbal communication. 
By summing up, this study will enable the researchers and practitioners to know the degree of extent of sustained eye contact developed under the structured format of discrete trial training program on one hand and provide the best fitted context for developing sustained eye contact in children with ASD on other hand. The study will also enable the special educationists and practitioners to introduce discrete trial training program and execute it inside the premises of govt. special education centers of the province of Punjab in the best fitted context for the betterment of this special segment of population with ASD.

\section{Literature Review}

Autism spectrum disorder (ASD) is a neuro-developmental disorder. It is a complex heterogeneous group of symptoms. Unresponsiveness, impaired understanding of social cues, intense focus on any object and repetitive or selfabusive behavior (s) are the core symptoms of this disorder. It basically affects social communication and behavior of children with varying degree of deficits (American Psychiatric Association, 2013).

Verbal and non-verbal social communication deficits is one of the hallmark features of children with ASD. Children with ASD have persistent deficits in social communication skills including verbal and non-verbal communication language (Cadette et al., 2016). According to (McEvoy et al., 1993) children with ASD show significantly fewer social interaction and joint attention skills as compare to the developmentally delayed children with the same non-verbal mental age and the normally developing children with the same verbal skills.

Is non-verbal behavior a deal of unsteady pattern of awareness and control (Knapp, 2013)? Lakin (2006) defined that non-verbal behaviors are encoded in brain with irregular and fluctuating degree of awareness and control. DePaulo and Friedman (1998) explained that non-verbal communication works to analyze perception on one hand and expression over perceived cue on other hand. However, children with ASD have marked deficits in non-verbal communication skills which entails that this segment of population lacks awareness and control to encode behaviors such as facial expressions, body posture, eye contact, gestures, proximity, touch and voice. As defined by Mehrabian (1972) that non-verbal communication is the subtle way to perceive and express ideas and feelings. But, this segment of population with ASD has persistent deficits to perceive and express ideas and feelings in multiple social contexts (American Psychiatric Association, 2013).

Eye contact is one of the types of non-verbal communication and the major impaired behavior found in children with ASD. Trevisanet al. (2017) explained that avoiding eye contact is the early indicator of this neuro-developmental disorder on one hand and elaborated that persistent difficulties of this behavior are to be faced throughout the lifespan of this segment of population. Falck-Ytter et al. (2015) defined that eye contact regulates cognitive processing system atypically in children with ASD. Senju and Johnson (2009) illustrated that unusual pattern of eye contact is 
the most significant symptom found in children with ASD. Madipakkam et al. (2017) also defined that deviant pattern to direct gaze is the hallmark feature of this segment of population.

However, children with ASD need to be intervened to communicate socially in an appropriate way. Mundy and Crowson (1997) defined that intensive and systematic early interventions lead to gains in developing joint attention skills and early social communication skills in children with ASD. Krstovska-Guerrero and Jones (2013) taught smiling coordinated with gaze behavior to respond to joint attention offers in children with ASD. Cook et al. (2017) used sequential model and showed notable gains in developing eye contact in children with ASD. Fonger and Malott (2019) employed shaping to develop eye contact for three seconds of duration in three participants of pre-school aged diagnosed with autism spectrum disorder. Jennett et al. (2008) explained that eye contact in three participants was a bit better under the format of discrete trial instruction.

Nosignificant literature is available on the topic in Pakistani society to support the researchers in order to find the gap and further to extend it. Therefore, the researchers planned to execute DTT to develop social communication skills in an individual with ASD. Therefore, the aim of the study was to manipulate DTT variable over developing social communication among children with ASD skills taken as dependent variable in order to investigate its efficacy in Pakistani society and ultimately to contribute to the field.

\section{Materials and Methods}

\section{Participant}

An adolescent girl with 15 years and 8 months diagnosed with autism spectrum disorder was chosen as the participant of the study.Participant A (a pseudonym) was enrolled at govt. special education, Gojra, Toba Tek Singh district of the province of Punjab, Pakistan. Participant Ahad no training of any type under any program since enrolled there. The participant was the minimal verbal adolescent with ASD and required support (level 1 of severity) observed on DSM-5 by the psychologist.

\section{Nature of Study}

This was quantitative research in its nature. It enabled the researchers to collect and analyze numerical data to interpret the obtained results.

\section{Research Design}

The phenomenon was experimented based on single subject study design coupled with interrupted time series design. This research design was consistent with specific reversal design (Seah, 1997) such as A-B-C-D-E-A and two follow up 
periods. A phase was the baseline. B phase, C phase, D phase, and $\mathrm{E}$ phase were the various intervention phases of discrete trial training and related conditions. The conditions or independent variable under which participants were required to respond are listed below in table 1 . The dependent variable was the number of correct responding over each stage of task analysis made by PA.

Table 1

Conditions across Phases in which Adolescent Girl was Required to Respond over Presented Cue

\begin{tabular}{|c|c|}
\hline Phases & Description \\
\hline A & Baseline (no treatment) \\
\hline B & $\begin{array}{l}\text { Administering treatment inside the classroom context and no error } \\
\text { correction }\end{array}$ \\
\hline $\mathrm{C}$ & Administering treatment inside the classroom context and error correction \\
\hline $\mathrm{D}$ & $\begin{array}{l}\text { Administering treatment inside the autism friendly training room and no } \\
\text { error correction }\end{array}$ \\
\hline $\mathrm{E}$ & $\begin{array}{l}\text { Administering treatment inside the autism friendly training room and error } \\
\text { correction }\end{array}$ \\
\hline A & Return to baseline (withdrawing treatment procedures) \\
\hline Follow up 1 & $\begin{array}{l}\text { Investigating appropriateness of the skill of sustained eye contact under } \\
\text { novel stimuli }\end{array}$ \\
\hline Follow up 2 & Generalization of the skill of sustained eye contact \\
\hline
\end{tabular}

Table 1 shows conditions across phases in which child was required to respond over presented cue.

\section{Instrumentation}

The researchers used self-developed research instruments to answer the formulated research questions of the study. Rating scale for the identification of social communication skills based on diagnostic and statistical manual of mental disorders (DSM-5) was developed and validated by experts to identify prior area of deficit of the chosen subject of the study. Experimental data was collected on DTT record keeping sheets for baseline measurements, treatment phases and follow up sessions.

\section{Procedures}

The researchers performed six steps in order to accomplish the requirements of the study. At first step, informed consent forms for the parents and teacher were designed to fulfill the research protocols. As participant A showed her consent to participate in the study but as participant $A$ was under the age of sixteen years; that is why the researchers had to obtain consent from the parents of the participant $A$ to make participation confirmed in the study. It was assured to the parents and teacher that there will be no harm during implementing discrete trials. Furthermore, it was assured to the parents and teacher that their names and other information related to 
their consent will be kept confidential and used for the purpose of the current research. In this way, an adolescent girl diagnosed with ASD was chosen to intervene under the formulated design of discrete trial training program.

After that, the researchers wrote permission letter and got permission from the headmistress of the Govt. Special education Center, Gojra, Toba Tek Singh to start the experiment upon the chosen participant of the study. Experiment was held during the timings of the Govt. Special education Center, Gojra, Toba Tek Singh. In this way, the researchers fulfilled the research ethics to run the study there.

At second step, self-developed rating scale was used to identify social communication deficits of the chosen subject of the study. This rating scale was provided to the class teacher of the subject of the study in order to fill it on the basis of observation. Impaired eye contact was identified as prior area of social communication deficit. The researchers then formulated the task objective surrounded by the prior area of deficit.

At third step, task objective based on the identified area of social communication deficit was formulated i.e. developing sustained eye contact. The child was expected to develop sustained eye contact for 2 to 3 seconds with most to least prompt ending at $90 \%$ correct responding across 3 consecutive DTT sessions. Mastery for each step is set at $90 \%$ correct independent responses during three consecutive teaching periods. Later, task analysis was performed to achieve the formulated task objective.

The researchers analyzed the task objective and divided it into 3 achievable steps as the following:

Step 1: The child will give a brief look (conscious glance) at conversational/communicative partner on producing table beat at right side of the subject during $90 \%$ correct responding across 3 consecutive DTT sessions.

Step 2: The child will give a steady and intent look (stabilized gaze) at conversational/communicative partner on producing table beat at left side of the subject during $90 \%$ correct responding across 3 consecutive DTT sessions.

Step 3: The child will realigning the line of sight towards the same object (gaze shift) at conversational/communicative partner on producing table beat once at right side and once at left side of the subject during $90 \%$ correct responding across 3 consecutive DTT sessions.

At fourth step, baseline phase was conducted over dependent variable (sustained eye contact) in order to set initial proficiency of the given task. It was conducted by the researchers in an empty classroom inside the premises of the Govt. Special education Centre, Gojra, Toba Tek Singh district of the Punjab province of Pakistan but out of the classroom of the chosen subject of the study. Later, four 
treatment phases were conducted based on intervention steps given by Smith (2001).These sessions were conducted by the researchers inside the classroom and autism friendly training room (temporarily designed) at the Govt. Special Education Centre Gojra. Amongst these treatment phases, phase B was conducted between participant and researcher inside the classroom context under no error correction technique to encourage correct responding over developing sustained eye contact; phase $\mathrm{C}$ was conducted between participant and researcher again inside the classroom context but under error correction technique over developing sustained eye contact; phase D was conducted between participant and researcher inside the autism friendly training room context (temporarily designed)under no error correction technique to encourage correct responding over developing sustained eye contact; and phase $\mathrm{E}$ was conducted between participant and researcher again inside the autism friendly training room context (temporarily designed) but under error correction technique over developing sustained eye contact for the chosen subject of the study. Later, no treatment phase was conducted and return to phase A was observed. Return to phase A was again conducted at the same classroom where the researchers set the steady state baseline phase to measure the return to phase A. Two follow up periods were observed such as first follow up period was conducted two weeks after observing the return to phase A and second follow up period was conducted after four weeks after observing the first follow up period. First follow up sessions were again conducted at the same classroom where the researchers set the steady state baseline phase to investigate the appropriateness of the developed skill of sustained eye contact under presenting novel stimuli. Second follow up sessions were again conducted at the same classroom where the researchers set the steady state baseline phase to generalize the results but in this time trial delivery authority was the class teacher (different but familiar person) of the chosen subject of the study.

The researchers set the following scheme of experimental sessions to carry the phases of the study out to reach the conclusions. Consequent upon that, the chosen subject of the study observed three experimental sessions each day spreading over 18 days of the discrete trial training program. There were 10 baseline sessions. Each baseline session was expanded over 2 minutes of duration to deliver the discriminative stimulus and to set the steady state initial proficiency level. 2 sessions were conducted each day across the baseline phase. In this way, 5 days were allocated to set the baseline phase. There were total 53 experimental sessions. Each experimental session was comprised of 3 DTT sessions. Each DTT session was containing 30 discrete trials. Each experimental session was spreading over 24 minutes of duration. 3 minutes were gives as break time after completing each DTT session within an experimental session. Each DTT session was expanded over 4 minutes of duration. 1 minute was given as break time. In this way each DTT session was expanded over 5 minutes of duration. 6 minutes were given as intra break time for starting each experimental session. Consequently, there were 10 return to phase A sessions and 20 follow up sessions, 10 sessions for each follow up period which 
was expanded over 2 minutes of duration to deliver the discriminative stimulus and to set the durability level of the discrete trial training program.

At fifth step, treatment integrity was observed on $100 \%$ session across all the phases while inter-observer reliability was measured for $50 \%$ randomly chosen sessions across all the phases of the discrete trial training program. It was calculated by the speech therapist of the chosen subject of the study.

At sixth step, which is the end, triad analysis was run to interpret the results. Descriptive analysis was performed to calculate the percentages of skill development across all the treatment phases. Visual analysis was performed to investigate the rate of change of the skill development across all the treatment phases. Likewise, tau-u analysis was performed to evaluate the effect size of the treatment across all the phases of the discrete trial training program.

\section{Data Analysis}

In this section, the researchers analyzed the obtained data and presented results of the study. The analysis was performed to support the results and ultimately satisfy the research questions of the study. Figure 1presents number of correct responding of sustained eye contact across experimental sessions.

\section{Figure 1}

Number of Correct Responding of Sustained Eye Contact across Experimental Sessions

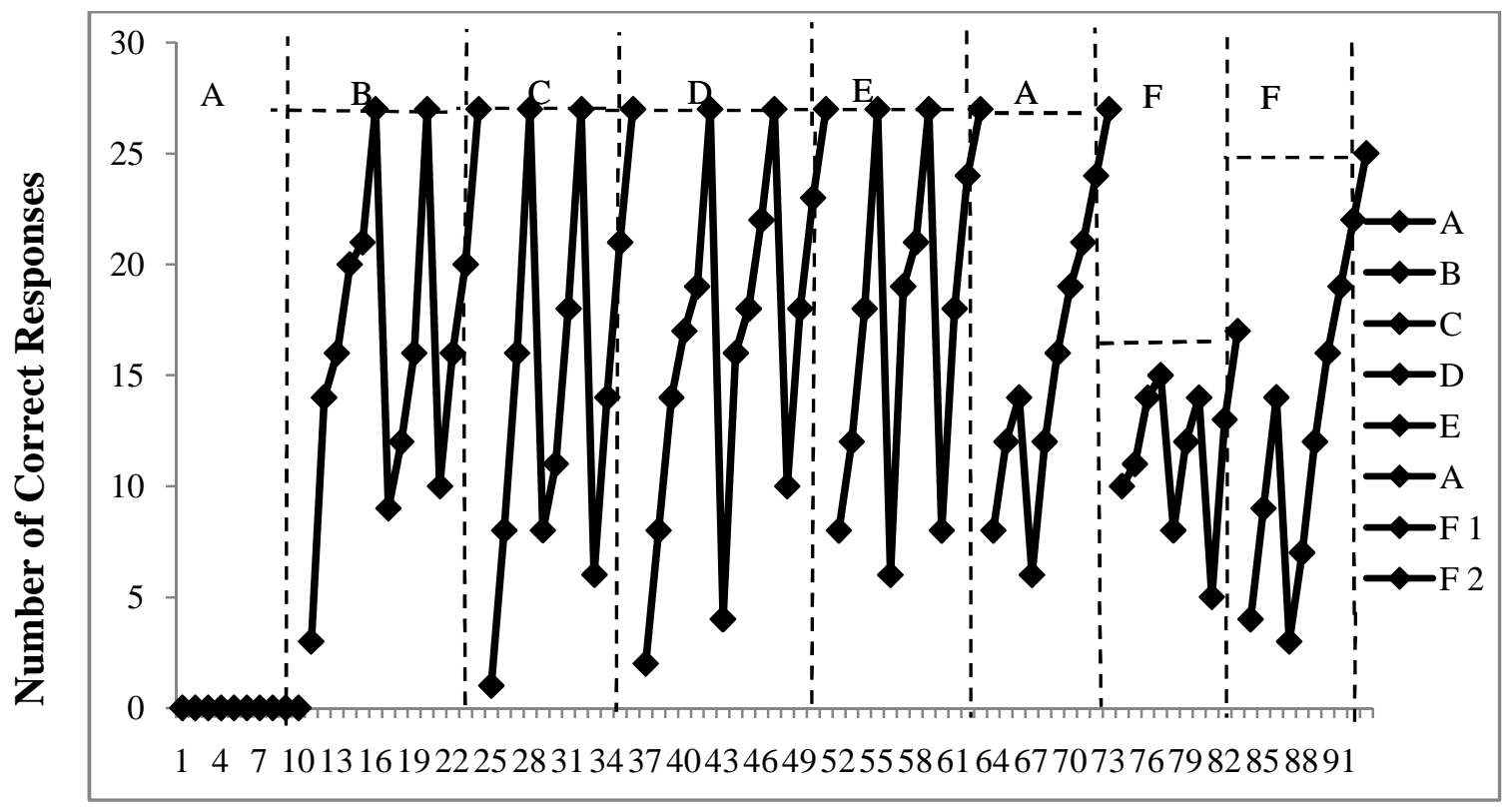

Sessions 
Figure 1 illustrates $51.1111 \%, 56.6666 \%, 56.00 \%$, and 59.72222\% development of eye contact across BCDE phases respectively. The trend of progress shows inconsistency throughout the phases with an ascending and/or descending order of achievement across the experimental sessions. The slopes in phase $\mathrm{C}$ and phase $\mathrm{E}$ show lower variability and irregular patterns of fluctuations around the mean slop as compare to phase B and phase D under the lower initial proficiency levels of presenting correct number of responses of sustained eye contact across all the phases of the experiment. The researchers measured tau $=0.00$ significant at $p=1.000$ across baseline phase; tau $=0.691$ significant at $p=0.000$ with medium to high size effect across $B$ phase; tau $=0.812$ significant at $p=0.000$ with medium to high size effect across $C$ phase; tau $=0.773$ significant at $p=0.000$ with medium to high size effect across $D$ phase; tau $=0.814$ significant at $p=0.000$ with medium to high size effect across $E$ phase; tau $=0.833$ significant at $p=0.000$ with medium to high size effect across A phase; tau $=0.833$ significant at $p=0.000$ with medium to high size effect across F1 phase and tau 0.830 significant at $p=0.000$ with medium to high size effect across F2 phase.

\section{Treatment Integrity}

Extent of treatment integrity was observed 100\% across all the treatment phases of the discrete trial training program.

\section{Inter-observer Reliability}

Inter-observer reliability was observed $86.2 \%$ between the researchers and the speech therapist of the chosen subject of the study.

\section{Discussion}

The purpose of the study was to investigate the efficacy of discrete trial training program in sustained eye contact in an adolescent girl diagnosed with autism spectrum disorder. Self-developed instruments enabled the researchers to gather data and analyze it later to interpret the results. Two research questions were formulated to answer in the current research. Each question is discussed below:

Question 1: Can sustained eye contact be developed by using discrete trails in an adolescent girl with autism spectrum disorder inside the premises of govt. special education centers of the province of Punjab, Pakistan?

Yes, sustained eye contact can be developed using discrete trials in children with autism spectrum disorder inside the premises of govt. special education centers of the province of Punjab, Pakistan. It is clear by figure 1 that all the treatment conditions encouraged the chosen subject of the study to respond over presented discriminative stimuli correctly. No doubt, progress to achieve mastery criteria was inconsistent but the maximum opportunities provided under the discrete trial 
training program enabled the chosen subject of the study to reach the predetermined task objective.

The study of the literature also supports the results of the current study as Klein et al. (2009) taught gaze following behavior in four years' old 3 participants under progressively prompt delay manner. The study as Krstovska-Guerrero and Jones (2013) taught smiling coordinated with gaze behavior to respond to joint attention offers in children with ASD. The study as Cook et al. (2017) used sequential model and showed notable gains in developing eye contact in children with ASD. The study conducted by Fonger and Malott(2019) also supports the results of the current study as these researchers employed shaping to develop eye contact for three seconds of duration in three participants of pre-school aged diagnosed with autism spectrum disorder.

More specifically, Jennett et al. (2008) concluded that eye contact in three participants was a bit better under the format of discrete trial instruction. The study conducted by Hamdan (2018) proposed the discrete trial training program to improve non-verbal communication skills including developing eye contact which deliberately supports the results of the current study.

Question 2: Which of the context is best fitted for developing sustained eye contact in children with autism spectrum disorder inside the premises of govt. special education centers of the province of Punjab, Pakistan?

This was a comparative question to answer. For this, four contexts were designed and analyzed in four treatment phases of the study. The best fitted context found as a result of error correction inside the autism friendly training room (temporarily designed) which was executed in phase $\mathrm{E}$ of the treatment.

Here, it is important to discuss that context matters less to develop sustained eye contact under the structured format of discrete trial training program. Contrary to this, the thing that matters is the formulation of discrete trials for providing enough opportunities to develop the skill such as sustained eye contact and/or other (s) social communication skills in children with ASD.

\section{Conclusions}

The researchers concluded based on the obtained results that the children with autism spectrum disorder can learn social communication skill under any context because each context appears new to them and they need to struggle with making adjustments necessary to pick social cues and to respond over them on one hand. On other hand, it is proved that autism friendly training room with error correction has comparatively higher importance to drive this segment of population with ASD towards achieving set objectives over other specifically designed contexts. Shortly, number of discrete trials matter instead of specifically designed contexts to develop social communication skill in this segment of population. 


\section{Recommendations}

The study recommends that furnished autism friendly training rooms should be established inside the premises of govt. special education centers across the Punjab province of Pakistan by the Punjab government of Pakistan in order to gain maximum advantages of introducing and executing the structured discrete trial training program for the betterment of this special segment of population with autism spectrum disorder.

\section{Implications}

The study will be helpful for special educationists and practitioners to introduce this strategy by implementing and executing discrete trial training program inside the premises of govt. special education centers of the province of Punjab in the best fitted context for the betterment of this special segment of population with ASD. The study may influence existing system of dealing children with ASD inside the premises of Govt. Special Education Centers/Schools across the province of Punjab, Pakistan. 


\section{References}

American Psychiatric Association. (2013). Diagnostic and statistical manual of mental disorders (5thed.). Washington, DC: Publisher.

Cadette, J. N., Wilson, C. L., Brady, M. P., Dukes, C., \& Bennett, K. D. (2016). The effectiveness of direct instruction in teaching students with autism Spectrum disorder to answer "wh-" questions. Journal of autism and developmental disorders, 46(9), 2968-2978.

Camp, W. (2001). Formulating and evaluating theoretical frameworks for career and technical education research. Journal of Vocational Education Research, 26(1), 4-25.

Cook, J. L., Rapp, J. T., Mann, K. R., McHugh, C., Burji, C., \&Nuta, R. (2017). A practitioner model for increasing eye contact in children with autism. Behavior Modification, 41(3), 382-404.

DePaulo, B. M., \& Friedman, H. S. (1998). Nonverbal communication, In D. T. Gilbert, S. T. Fiske, \& G. Lindzey (Eds.), The handbook of social psychology (p. 3-40). McGraw-Hill.

DeQuinzio, J., Townsend, D., Sturmey, P., \& Poulson, C. (2007). Generalized imitation of facial models by children with autism. Journal of Applied Behavior Analysis, 40(4), 755-759.

Engel, R. J., \& Schutt, R. K. (2013). The practice of research in social work (3rd ed.). Sage Publications, Inc.

Falck-Ytter, T., Carlström, C., \& Johansson, M. (2015). Eye contact modulates cognitive processing differently in children with autism. Child Development, 86(1), 37-47.

Fonger, A. M., \& Malott, R. W. (2019). Using shaping to teach eye contact to children with autism spectrum disorder. Behavior analysis in practice, 12(1), 216-221.

Garfinkle, A., \& Schwartz, I. (2002). Peer imitation increasing social interactions in children with autism and other developmental disabilities in inclusive preschool classrooms. Early Childhood Special Education, 22(1), 26-38.

Garcia-Albea, E., Reeve, S., Reeve, K., \& Brothers, K. (2014). Using audio script fading and multiple exemplar training to increase vocal interactions in children with autism. Journal of Applied Behavior Analysis, 47(2), 325-343.

Gena, A., Couloura, S., \&Kymissis, E. (2005). Modifying the affective behavior of preschoolers with autism using in-vivo or video modeling and reinforcement contingencies. Journal of Autism and Developmental Disorders, 35(5), 545-556. 
Groskreutz, M., Peters, A., Groskreutz, N., \& Higbee, T. (2015). Increasing playbased commenting inchildren with autism spectrum disorder using a novel script-frame procedure. Journal of Applied Behavior Analysis, 48(2), 442-447.

Hamdan, M. A. (2018). Developing a Proposed Training Program Based on Discrete Trial Training (DTT) to Improve the Non-Verbal Communication Skills in Children with Autism Spectrum Disorder (ASD). International Journal of Special Education, 33(3), 579-591.

Harris, S., Handleman, J., \& Alessandri, M. (1990). Teaching youths with autism to offer assistance. Journal of Applied Behavior Analysis, 23(3), 297-305.

Jennett, H. K., Harris, S. L., \&Delmolino, L. (2008). Discrete trial instruction vs. mand training for teaching children with autism to make requests. The Analysis of Verbal Behavior, 24(1), 69-85.

Jones, E., Carr, E., \&Feeley, K. (2006). Multiple effects of joint attention intervention for children with autism. Behavior Modification, 30(6), 782-834.

Kasari, C., Freeman, S., \& Paparella, T. (2006). Joint attention and symbolic play in young children with autism: A randomized controlled intervention study. Journal of Child Psychology and Psychiatry, 47(6),611-620.

Klein, J. L., MacDonald, R. P., Vaillancourt, G., Ahearn, W. H.,\& Dube, W. V. (2009). Teaching discrimination of adult gaze direction to children with autism. Research in autism spectrum disorders, 3(1), 42-49.

Knapp, M. L., Hall, J. A., \&Horgan, T. G. (2013). Nonverbal communication in human interaction. Cengage Learning.

Krstovska-Guerrero, I., \& Jones, E. A. (2013). Joint attention in autism: Teaching smiling coordinated with gaze to respond to joint attention bids. Research in Autism Spectrum Disorders, 7(1), 93-108.

Lakin, J. L. (2006). Automatic Cognitive Processes and Nonverbal Communication. In V. Manusov \& M. L. Patterson (Eds.), The Sage handbook of nonverbal communication (p. 59-77). Sage Publications, Inc. https:// doi.org/10.4135/9781412976152.n4

LeBlanc, L., Coates, A., Daneshvar, S., Charlop-Christy, M., Morris, C., \& Lancaster, B. (2003). Using video modeling and reinforcement to teach perspective taking skills to children with autism. Journal of Applied Behavior Analysis, 36(2), 253-257.

Madipakkam, A. R., Rothkirch, M., Dziobek, I., \& Sterzer, P. (2017). Unconscious avoidance of eye contact in autism spectrum disorder. Scientific reports, 7(1), 1-6. 
Marzullo-Kerth, D., Reeve, S., Reeve, K., \& Townsend, D. (2011). Using multipleexemplar training to teach a generalized repertoire of sharing to children with autism. Journal of Applied Behavior Analysis,22(2), 279-294.

McEvoy, R. E., Rogers, S. J., \& Pennington, B. F. (1993). Executive function and social communication deficits in young autistic children. Journal of child psychology and psychiatry, 34(4), 563-578.

Mehrabian, A. (1972). Nonverbal communication. Transaction Publishers.

Mundy, P., \&Crowson, M. (1997). Joint attention and early social communication: Implications for research on intervention with autism. Journal of Autism and Developmental disorders, 27(6), 653-676.

Nugent, W. (2009). Analyzing single system design data. Oxford University Press.

Schrandt, J., Townsend, D., \& Poulson, C. (2009). Teaching empathy skills to children with autism. Journal of Applied Behavior Analysis, 42(1), 17-32.

Seah, P. P. (1997). Increasing eye contact and appropriate verbalizations of young children with autistic characteristics. Cowan University

Reeve, S., Reeve, K., Townsend, D., \& Poulson, C. (2007). Establishing a generalized repertoire ofhelping behavior in children with autism. Journal of Applied Behavior Analysis, 40(1), 123-136.

Senju, A., Yaguchi, K., Tojo, Y., \& Hasegawa, T. (2003). Eye contact does not facilitate detection in children with autism. Cognition, 89(1), B43-B51.

Senju, A., \& Johnson, M. H. (2009). Atypical eye contact in autism: models, mechanisms and development. Neuroscience EBiobehavioral Reviews, 33(8), 12041214.

Smith, T. (2001). Discrete trial training in the treatment of autism. Focus on autism and other developmental disabilities, 16(2), 86-92.

Trevisan, D. A., Roberts, N., Lin, C., \& Birmingham, E. (2017). How do adults and teens with self declared Autism Spectrum Disorder experience eye contact? A qualitative analysis of first-hand accounts. PloS one, 12(11), e0188446. 